\title{
A randomised controlled study of high intensity exercise as a dishabituating stimulus to improve hypoglycaemia awareness in people with type 1 diabetes: a proof-of-concept study
}

\author{
Catriona M. Farrell ${ }^{1}$ - Alison D. McNeilly ${ }^{1} \cdot$ Paul Fournier $^{2} \cdot$ Timothy Jones $^{3} \cdot$ Simona M. Hapca $^{4}$ - Daniel West ${ }^{5}$. \\ Rory J. McCrimmon ${ }^{1}$
}

Received: 30 August 2019 / Accepted: 25 November 2019/Published online: 15 January 2020

(C) The Author(s) 2019

\begin{abstract}
Aims/hypothesis Approximately 25\% of people with type 1 diabetes have suppressed counterregulatory hormonal and symptomatic responses to insulin-induced hypoglycaemia, which renders them at increased risk of severe, disabling hypoglycaemia. This is called impaired awareness of hypoglycaemia (IAH), the cause of which is unknown. We recently proposed that IAH develops through habituation, a form of adaptive memory to preceding hypoglycaemia. Consistent with this hypothesis, we demonstrated restoration of defective counterregulatory hormonal responses to hypoglycaemia (referred to as dishabituation) in a rodent model of IAH following introduction of a novel stress stimulus (high intensity training [HIT]). In this proof-of-concept study we sought to further test this hypothesis by examining whether a single episode of HIT would amplify counterregulatory responses to subsequent hypoglycaemia in people with type 1 diabetes who had IAH (assessed by Gold score $\geq 4$, modified Clarke score $\geq 4$ or Dose Adjustment For Normal Eating (DAFNE) hypoglycaemia awareness rating 2 or 3 ). The primary outcome was the difference in adrenaline response to hypoglycaemia following both a single episode of HIT and rest.

Methods In this randomised, crossover study 12 participants aged between 18 and 55 years with type 1 diabetes for $\geq 5$ years and an $\mathrm{HbA}_{1 \mathrm{c}}<75 \mathrm{mmol} / \mathrm{mol}(9 \%)$ were recruited. Individuals were randomised using computer generated block randomisation to start with one episode of HIT $\left(4 \times 30\right.$ s cycle sprints [ 2 min recovery] at $150 \%$ of maximum wattage achieved during $\dot{V} \mathrm{O}_{2 \text { peak }}$ assessment) or rest (control). The following day they underwent a 90 min hyperinsulinaemic-hypoglycaemic clamp study at $2.5 \mathrm{mmol} / \mathrm{l}$ with measurement of hormonal counterregulatory response, symptom scores and cognitive testing (four-choice reaction time and digit symbol substitution test). Each intervention and subsequent clamp study was separated by at least 2 weeks. The participants and investigators were not blinded to the intervention or measurements during the study. The investigators were blinded to the primary outcome and blood analysis results.

Results All participants (six male and six female, age 19-54 years, median [IQR] duration of type 1 diabetes 24.5 [17.3-29.0] years, mean [SEM] $\mathrm{HbA}_{1 \mathrm{c}} 56$ [3.67] mmol/mol; 7.3\% [0.34\%]) completed the study (both interventions and two clamps). In comparison with the rest study, a single episode of HIT led to a $29 \%$ increase in the adrenaline (epinephrine) response (mean [SEM]) (2286.5 [343.1] vs 2953.8 [384.9] pmol/1); a significant increase in total symptom scores (Edinburgh Hypoglycaemia Symptom Scale: 24.25 [2.960 vs 27.5 [3.9]; $p<0.05)$, and a significant prolongation of four-choice reaction time (591.8 [22.5] vs $659.9[39.86] \mathrm{ms} ; p<0.01]$ during equivalent hypoglycaemia induced the following day.
\end{abstract}

Electronic supplementary material The online version of this article (https://doi.org/10.1007/s00125-019-05076-5) contains peer-reviewed but unedited supplementary material, which is available to authorised users.

Rory J. McCrimmon

r.mccrimmon@dundee.ac.uk

1 Division of Systems Medicine, School of Medicine, University of Dundee, Dundee DD19SY, UK

2 Faculty of Science, School of Human Science, The University of Western Australia, Perth, Australia
3 Faculty of Health and Medical Sciences, Paediatrics, The University of Western Australia, Perth, Australia

4 Computing Science and Mathematics, Faculty of Natural Sciences, University of Stirling, Stirling, UK

5 Institute of Cellular Medicine, Faculty of Medical Science, Newcastle University, Newcastle, UK 


\section{Research in context}

\section{What is already known about this subject?}

- People with type 1 diabetes have suppressed counterregulatory hormonal and symptom responses to hypoglycaemia as a result of recurrent exposure to hypoglycaemia; this is referred to as impaired awareness of hypoglycaemia (IAH)

- The mechanism underlying development of IAH is not fully understood but shows many features in keeping with the form of learned behaviour known as habituation

What is the key question?

- Does IAH develop as a form of habituation and can it therefore be reversed by introducing a novel stressor (dishabituation)?

What are the new findings?

- Our findings are consistent with the hypothesis that IAH develops as a habituated response

- We show improved hormonal, symptomatic and cognitive response to hypoglycaemia in people with type 1 diabetes who have $\mathrm{IAH}$, following dishabituation with a single period of high intensity exercise

How might this impact on clinical practice in the foreseeable future?

- If proven in longer-term clinical trials, high intensity exercise may represent a novel therapeutic intervention for people with type 1 diabetes and IAH

Conclusions/interpretation These findings are consistent with the hypothesis that IAH develops in people with type 1 diabetes as a habituated response and that introduction of a novel stressor can restore, at least partially, the adapted counterregulatory hormonal, symptomatic and cognitive responses to hypoglycaemia.

Trial registration ISRCTN15236211.

Keywords Behaviour $\cdot$ Counterregulation $\cdot$ Diabetes $\cdot$ Exercise $\cdot$ Habituation $\cdot$ Hypoglycaemia $\cdot$ Impaired awareness

$\begin{array}{ll}\text { Abbreviations } \\ \text { 4CRT } & \text { Four-choice reaction time } \\ \text { CGM } & \text { Continuous glucose monitor } \\ \text { DAFNE } & \text { Dose Adjustment For Normal Eating } \\ \text { DSST } & \text { Digit symbol substitution test } \\ \text { EHS } & \text { Edinburgh Hypoglycaemia Symptom Scale } \\ \text { HAAF } & \text { Hypoglycaemia-associated autonomic failure } \\ \text { HIT } & \text { High intensity training } \\ \text { IAH } & \text { Impaired awareness of hypoglycaemia }\end{array}$

\section{Introduction}

Severe hypoglycaemia is an adverse effect of insulin therapy in diabetes that has a well-recognised morbidity and mortality [1]. Fear of hypoglycaemia often outweighs concerns about longterm consequences of chronic hyperglycaemia [2], and acts as a barrier to patients achieving optimal glycaemic control [3]. The major risk factor for severe hypoglycaemia is impaired awareness of hypoglycaemia (IAH), which increases the risk of an event six-fold [4]. People with IAH have a reduced ability to perceive the onset of acute hypoglycaemia [5]. It affects $25 \%$ of all people with type 1 diabetes and, of concern, the incidence of IAH has not changed in the last 2-3 decades despite the introduction of insulin analogues and improved insulin delivery systems [6]. IAH is an acquired abnormality that should be placed alongside chronic microvascular complications such as retinopathy, neuropathy and nephropathy as it can be just as serious and disabling.

The average individual with type 1 diabetes will experience about two episodes of symptomatic hypoglycaemia per week $[7,8]$. Ordinarily, hypoglycaemia initiates a biological counterregulatory response that comprises a broad range of physiological, symptomatic and behavioural changes that together act to restore normal glucose homeostasis. Failure of this biological response leads to progressive hypoglycaemia and may result in individuals being unable to self-treat or to loss of consciousness (severe hypoglycaemia). In their seminal study in 1991, Heller and Cryer demonstrated that prior exposure to hypoglycaemia leads to suppression of both hormonal and symptomatic responses during hypoglycaemia of the same depth and duration induced the following day [9]. This finding 
was later confirmed in people with type 1 diabetes [10], and it was further established that increasing the frequency [11] and duration [12] of prior exposure to hypoglycaemia increases the extent of counterregulatory response suppression, while strict avoidance of hypoglycaemia restores counterregulatory responses [13]. Cumulatively, this exposure to recurrent hypoglycaemia leads to the development of IAH where individuals demonstrate a reduced magnitude and increased threshold (lower glucose) for sympathoadrenal activation and symptomatic experience of hypoglycaemia. Unfortunately, despite nearly three decades since Heller's and Cryer's seminal work, the biological mechanisms underpinning the development of IAH remain unknown.

Habituation is a form of adaptive memory that develops in many organisms in response to a repeated, often stressful stimulus [14]. Habituation has been defined as a reduction in the psychological, behavioural or physiological response to a stimulus as a result of recurrent or prolonged exposure [14, 15]. Individuals with type 1 diabetes who develop IAH demonstrate reduced physiological (hormonal and symptomatic), psychological (reduced anxiety) and behavioural (inappropriate responses to hypoglycaemia while driving) responses to hypoglycaemia [14-18]. This led us to propose that IAH may represent a habituated response to repeated hypoglycaemia. To test this hypothesis, we recently examined in a rodent model whether IAH could be reversed, at least temporarily, by the introduction of a single novel stress stimulus, a phenomenon referred to as dishabituation. Rodents were exposed to non-severe hypoglycaemia three times a week for a period of 4 weeks (habituation), and subsequently randomised to a single burst of high intensity training (HIT; dishabituation) or control (no exercise) before undergoing a controlled experimental hypoglycaemia study $24 \mathrm{~h}$ later [19]. In comparison with control animals, those who had been exposed to the dishabituatory stimulus had a significantly greater counterregulatory hormonal response during the controlled hypoglycaemia study [19]. While this finding supported the hypothesis that IAH develops as a result of habituation, the rodents did not have type 1 diabetes and the counterregulatory response was assessed via hormonal measures only [19]. In the current proof-of-concept study, we sought to further test the habituation hypothesis by examining whether a single burst of HIT would act as a dishabituatory stimulus in people with long-duration type 1 diabetes who had IAH.

\section{Methods}

This was a single-centre, randomised crossover study carried out at Ninewells Hospital, Dundee, Scotland. Ethics approval was obtained from an independent research ethics committee $(17 / \mathrm{SS} / 0150)$ and the study was registered with the
International Standard Randomised Controlled Trials Register (ISRCTN15236211). The study was conducted in accordance with the Declaration of Helsinki, and written informed consent was obtained from all participants before inclusion in the study.

Inclusion criteria were: diagnosis of type 1 diabetes for at least 5 years, treatment with multiple daily injections or continuous subcutaneous insulin infusion, $\mathrm{HbA}_{1 \mathrm{c}}$ $<75 \mathrm{mmol} / \mathrm{mol}(9 \%)$, age of 18 to 55 years, and IAH (Gold score [4] $\geq 4$, modified Clarke score [20] $\geq 4$ or Dose Adjustment For Normal Eating [DAFNE] hypoglycaemia awareness rating [21] 2 or 3). Exclusion criteria were: history of significant cardiac, renal, respiratory or neurological disease, pregnancy or breastfeeding, treatment with betablockers, history of high-risk foot disease, competitive sportsman [22] or participation in HIT in the preceding 6 months, or physical disability that might limit exercise.

The randomisation relates to the order that the HIT or rest intervention was carried out by the participant. Randomisation was carried out in a good clinical practice (GCP)-compliant manner using http://www.randomization.com/.

Participants were identified using the Scottish Diabetes Research Network (SDRN) and from the diabetes outpatient clinics in NHS Tayside, Scotland. The study took place at the Clinical Research Centre, Ninewells Hospital and Medical School, Dundee.

After obtaining informed written consent and initial screening, which included confirmation of IAH, each participant attended the Clinical Research Centre on seven separate occasions (See Fig. 1). During visit 1 anthropometric measurements, physical examination and electrocardiogram were performed. In addition, participants undertook an incremental maximal to volitional exhaustion exercise test using on a cycle ergometer (Corival, Lode, Groningen, the Netherlands; Metamax 3B, CORTEX Biophysik, Leipzig, Germany) to determine their $\dot{V} \mathrm{O}_{2}$ and peak heart rate.

For this study we have defined HIT as episodic short bursts of high intensity exercise separated by short periods of recovery at lower intensity [23]. The HIT programme used in this study was $20 \mathrm{~min}$ in duration; 5 min of gentle warm up cycling at $50 \mathrm{~W}, 40-60 \mathrm{rev} / \mathrm{min}$ followed by $4 \times 30 \mathrm{~s}$ cycle sprints [2 min recovery] at $150 \%$ of maximum wattage achieved during $\dot{V} \mathrm{O}_{2 \text { peak }}$ assessment with an aim to achieve $\geq 90 \%$ peak heart rate; concluding with $5 \mathrm{~min}$ cool down at $50 \mathrm{~W}, 40$ $60 \mathrm{rev} / \mathrm{min}$. Standardised advice [24] was given regarding blood glucose levels and insulin dose adjustment prior to and following HIT. The control intervention was $20 \mathrm{~min}$ of seated rest. Venous blood samples were taken for counterregulatory hormones (adrenaline, noradrenaline [norepinephrine], glucagon and lactate) at baseline of both the rest and HIT intervention visits with samples also taken after the third and fourth bouts of exercise during the HIT intervention. Each participant underwent a single HIT programme or controlled rest intervention the afternoon 
Fig. 1 Study flow diagram showing the different stages of the study

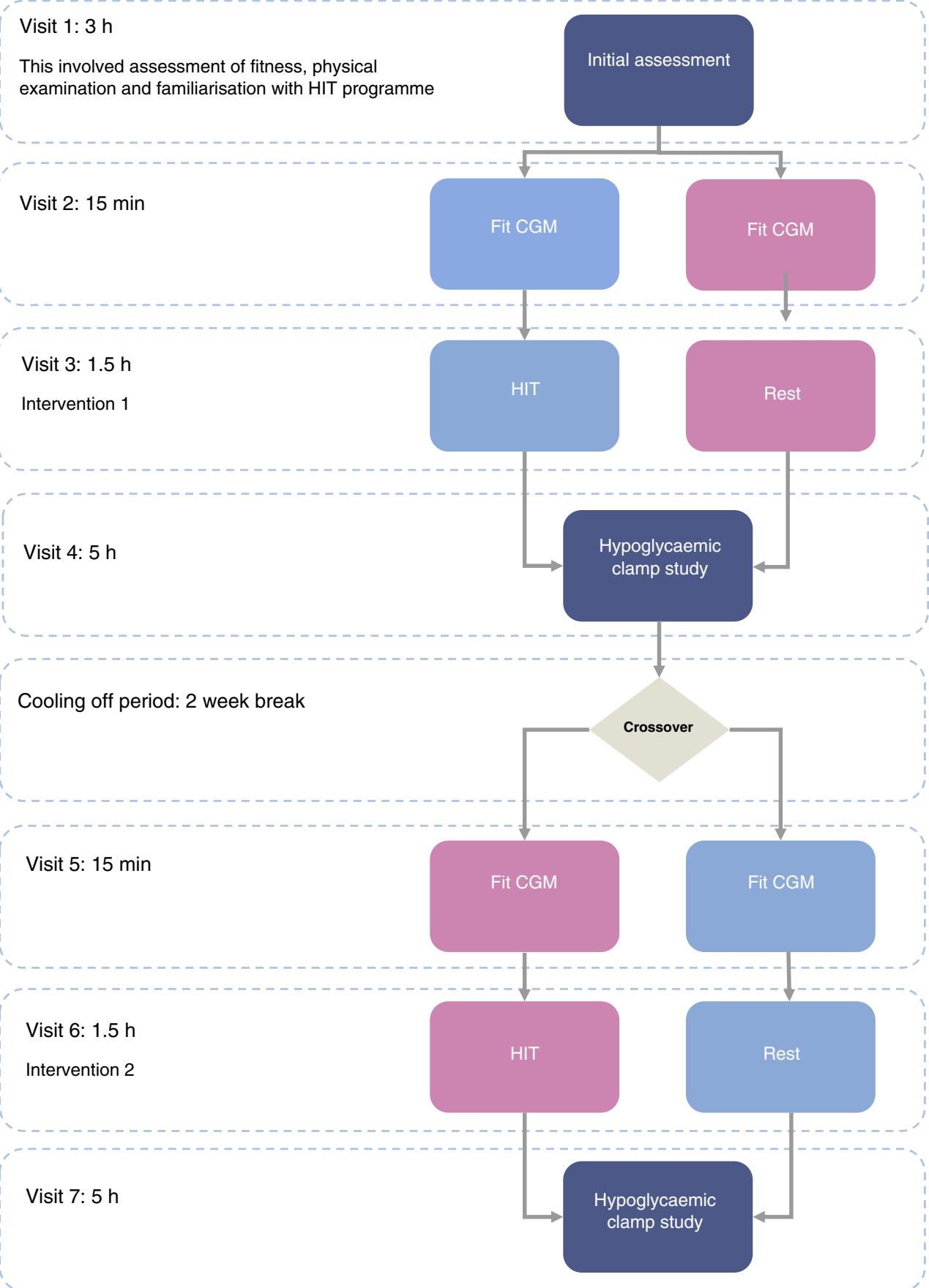

before undergoing a hyperinsulinaemic-hypoglycaemic clamp study the following day. The hyperinsulinaemichypoglycaemic clamp study was used to assess their hormonal counterregulatory response, symptom awareness and cognitive response. Hyperinsulinaemic-hypoglycaemic clamp study is considered the gold standard for comparing counterregulatory response to equivalent hypoglycaemia [25]. There was a 2 week cooling off period between the interventions to avoid any lasting effect prior to crossing in to the second arm of the study.
Experimental hypoglycaemia For each of the two clamp study visits (separated by at least 2 weeks) participants initially attended to be fitted with a real-time continuous glucose monitor (CGM) (Dexcom G4, Dexcom, San Diego, CA, USA), with low glucose alarm (set at $4.0 \mathrm{mmol} / \mathrm{l}$ ), at least $48 \mathrm{~h}$ before the study, to ensure absence of significant hypoglycaemia ( $<3 \mathrm{mmol} / 1$ for $>20 \mathrm{~min}$ ) [26] before the clamp procedure.

The evening preceding the clamp study, participants were advised to reduce their night-time basal insulin by $\approx 20 \%$ and fast for at least $8 \mathrm{~h}$ before coming to the Clinical Research 
centre at 08:00 hours. On the morning of the clamp, a retrograde cannula was inserted into a dorsal vein of the nondominant hand. It was then placed in a heated box at 55$60^{\circ} \mathrm{C}$ to arterialise venous blood. This line was used for blood sampling during the clamp study. In the contralateral arm, the antecubital vein was cannulated and used for insulin and dextrose infusions.

Prior to commencing the hypoglycaemic clamp, CGM data were analysed to ensure there was no hypoglycaemia in the preceding $24 \mathrm{~h}$. If there was evidence of hypoglycaemia the study was postponed and the intervention visit repeated. The study was postponed and intervention repeated three times in total by two participants: one participant had to repeat both interventions and one had to repeat the rest intervention alone.

The insulin infusion $(0.3 \mathrm{U} / \mathrm{ml})$ was started at a rate of $50 \mathrm{ml} / \mathrm{h}$ for priming purposes until the blood glucose dropped to below $7 \mathrm{mmol} / \mathrm{l}$ into the euglycaemic range, after which a rate of $1.5 \mathrm{mU}^{-1} \mathrm{~kg}^{-1} \mathrm{~min}^{-1}$ was maintained for the duration of the clamp. $20 \%$ dextrose was simultaneously infused at a variable rate (Infusomat Space, B. Braun Medical, Sheffield, UK) based on frequent (every $5 \mathrm{~min}$ ) bedside plasma glucose measurements (Biosen CLine GP+, EKF diagnostics, Cardiff, UK) to maintain blood glucose at pre-determined levels. Euglycaemia (glucose 4-6 mmol/l) was achieved and maintained for the first $30 \mathrm{~min}$ of the glucose clamp study, and subsequently, blood glucose was reduced over $30 \mathrm{~min}$ to a final glucose level of $2.5 \mathrm{mmol} / \mathrm{l}$. Blood glucose was maintained at $2.5 \mathrm{mmol} / 1$ for $60 \mathrm{~min}$ before glucose levels were returned to the euglycaemic range.

Counterregulatory hormones Arterialised blood for insulin and counterregulatory hormones (adrenaline, noradrenaline, glucagon and lactate) was taken every $30 \mathrm{~min}(t=-30,0$, 30, 60 and $90 \mathrm{~min}$ ) during the clamp.

Symptoms At each 30 min time point, participants completed a validated questionnaire, the Edinburgh Hypoglycaemia Symptom Scale (EHS) [27], scoring 11 symptoms from 1 (not at all) to 7 (very severe) on a visual analogue scale.

Cognitive function tests A series of psychometric tests recognised to be sensitive to hypoglycaemia were carried out in the same order, starting approximately $2 \mathrm{~min}$ before each 30 min time point: digit symbol substitution test (DSST) [28] and four-choice reaction time (4CRT) [29].

Laboratory assays Arterialised blood samples were taken every 5 min during the clamp and centrifuged, and plasma glucose was analysed at the bedside by an enzymaticamperometric method using chip-sensor technology (Biosen $\mathrm{C}$-Line GP+). Samples taken at $30 \mathrm{~min}$ intervals were centrifuged within $1 \mathrm{~h}$ to separate the serum or plasma and stored at $-80^{\circ} \mathrm{C}$ before assay. Hormone levels of insulin (Alpco, Salem, NH, USA; CV inter 4.9\%, intra 7.2\%), adrenaline (Alpco; CV inter 19.6\%, intra 14.3\%), noradrenaline (Alpco; CV inter $16.3 \%$, intra $12.0 \%$ ), glucagon (EMD Millipore, Billerica, MA, USA; CV inter $9.8 \%$, intra $6.52 \%$ ) and lactate (Siemens Advia 2100, Siemens Healthcare Diagnostics, Tarrytown, NY, USA) were measured, and samples were analysed in duplicate according to the manufacturer's instructions.

Data and statistical analysis The predefined primary endpoint was the difference in adrenaline response at a glucose level of $2.5 \mathrm{mmol} / \mathrm{l}$ following rest and HIT. Power calculations were based on a previous hypoglycaemic clamp study performed in a similar cohort of participants with type 1 diabetes and IAH conducted in our laboratory [30]. For a matched analysis, ten participants were required to detect a difference of $1 \mathrm{SD}$ with $\alpha$ set at 0.05 and $80 \%$ power. Twelve participants were recruited to allow for any participants who did not complete both experimental studies. Secondary outcomes examined were: difference in symptom awareness score, cognitive function and other counterregulatory hormone responses during hypoglycaemia $(2.5 \mathrm{mmol} / \mathrm{l})$ following rest and HIT intervention. For the primary and secondary endpoints, a generalised estimated equation was used adjusting for order effect and baseline as covariates. A $p$ value $<0.05$ was considered statistically significant. Paired $t$ tests were used to assess hormonal and heart rate response to HIT. All data are presented as mean \pm SEM. Statistical analyses were conducted using IBM SPSS Statistics 22 software, IBM, Armonk, NY, USA.

\section{Results}

Participant characteristics Recruitment ran from January 2018 to August 2018. Of the 18 participants screened, five did not meet the inclusion criteria and one was withdrawn; 12 participants gave informed written consent and were randomised (see Fig. 2). The 12 participants (six male and six female, aged 19-54 years) who completed the study had long-duration type 1 diabetes (median [IQR] duration of type 1 diabetes 24.5 [17.3-29.0] years] and IAH defined by at least one of a Gold score $\geq 4$ (7/12 participants), modified Clarke] score $\geq 4$ (10/12 participants) or DAFNE hypoglycaemia awareness rating 2 or 3 (11/12 participants) (electronic supplementary material [ESM] Table 1). Mean (SEM) $\mathrm{HbA}_{1 \mathrm{c}}$ at randomisation was $56(3.67) \mathrm{mmol} / \mathrm{mol} ; 7.3 \%(0.34)$.

Physiological response to HIT in type 1 diabetes with IAH All participants were able to complete the HIT intervention which involved $4 \times 30$ s cycle sprints ( 2 min recovery) at $150 \%$ of 


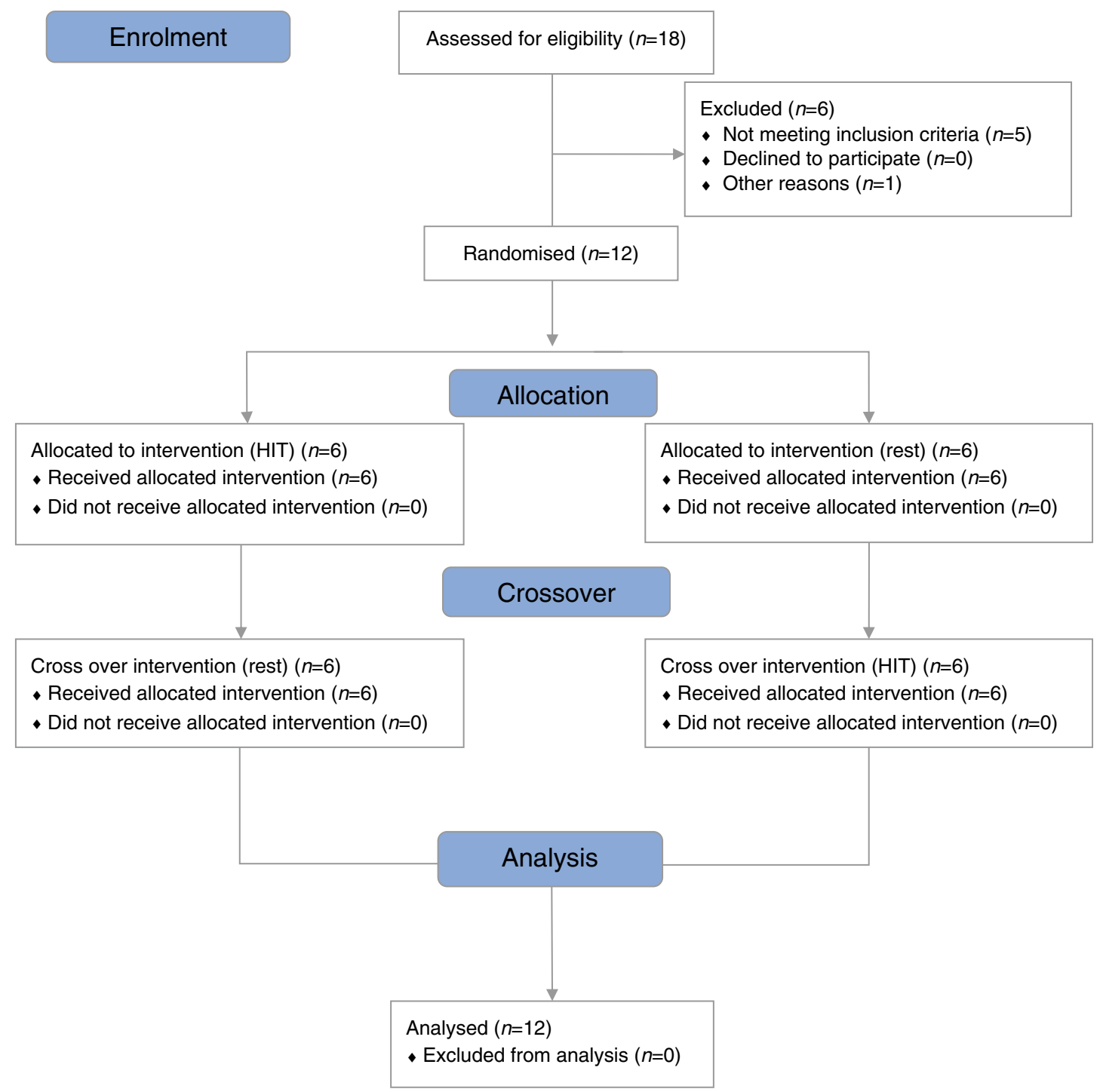

Fig. 2 Consort diagram showing participant allocation at each stage of the study

maximum wattage achieved during $\dot{V} \mathrm{O}_{2 \text { peak }}$ assessment. Each participant achieved their target $>90 \%$ peak heart rate (Fig. 3a). The HIT programme provided an intense stimulus to adrenaline (mean [SEM] 228.45 [54.65] vs 1236.83 [203.42] $\mathrm{pmol} / \mathrm{l}$; rest vs HIT, $p<0.001]$ (Fig. 3b) and noradrenaline (4.77 [0.55] vs 20.42 [2.28] pmol/1; $p<0.001]$ (Fig. 3c), and resulted in a marked 12 -fold increase in lactate $(0.95[0.09]$ vs $12.28[0.78] \mathrm{mmol} / \mathrm{l} ; p<0.001$ ) (Fig. 3d).

Dishabituation with HIT in type 1 diabetes with IAH The day following HIT or rest interventions, participants underwent a hyperinsulinaemic-hypoglycaemic clamp to assess their counterregulatory response. During the clamp, plasma glucose profiles were well matched (Fig. 4a) at baseline (mean [SEM] $4.81[0.06]$ vs $4.80[0.07] \mathrm{mmol} / 1$ [rest vs HIT]) and at steady state hypoglycaemia (30-90 $\mathrm{min})(2.65[0.06]$ vs 2.54 $[0.06] \mathrm{mmol} / \mathrm{l} ; p=0.23)$. Mean glucose infusion rates $(30$ -
$90 \mathrm{~min})$ were lower following HIT (2.80 [0.10] vs 3.07 [0.12]), but this difference was not significant; $p=0.23$ (Fig. 4b). Plasma insulin was maintained at $704.1(17.2)$ vs 655.3 (17.2) $\mathrm{pmol} / \mathrm{l}$ throughout the clamp period $(p=0.30)$.

Hormonal counterregulatory response The single HIT intervention had an overall significant effect on the adrenaline response to subsequent hypoglycaemia. Mean (SEM) 90 min hypoglycaemia-induced adrenaline following rest and HIT interventions, respectively, was 2286.5 (343.1) vs 2953.8 (384.9) pmol/1; $p<0.05$ (Fig. 5a). A smaller, nonsignificant rise in noradrenaline (5308.3 [656.5] vs 5754.8 [771.2] pmol/l) also followed HIT $(p=0.196)$. Interestingly, although not of statistical significance, there was a small increase in glucagon during equivalent hypoglycaemia following HIT (48.7 [6.7] vs 57.1 [9.1] ng/l; $p=0.238$; Fig. 5b). 
Fig. 3 Physiological responses to HIT for each participant. (a) Heart rate during rest, at the $90 \%$ maximum heart rate target determined from the $\dot{V} \mathrm{O}_{2 \text { peak }}$ test, and the maximum heart rate reached during HIT. (b, c, d) Adrenaline (b), noradrenaline (c) and lactate (d) during rest and HIT interventions. $n=12$; values are the mean \pm SEM. $* * * p<$ 0.001 by paired $t$ test comparing rest with HIT or max HR reached. $\mathrm{HR}$, heart rate; max, maximum
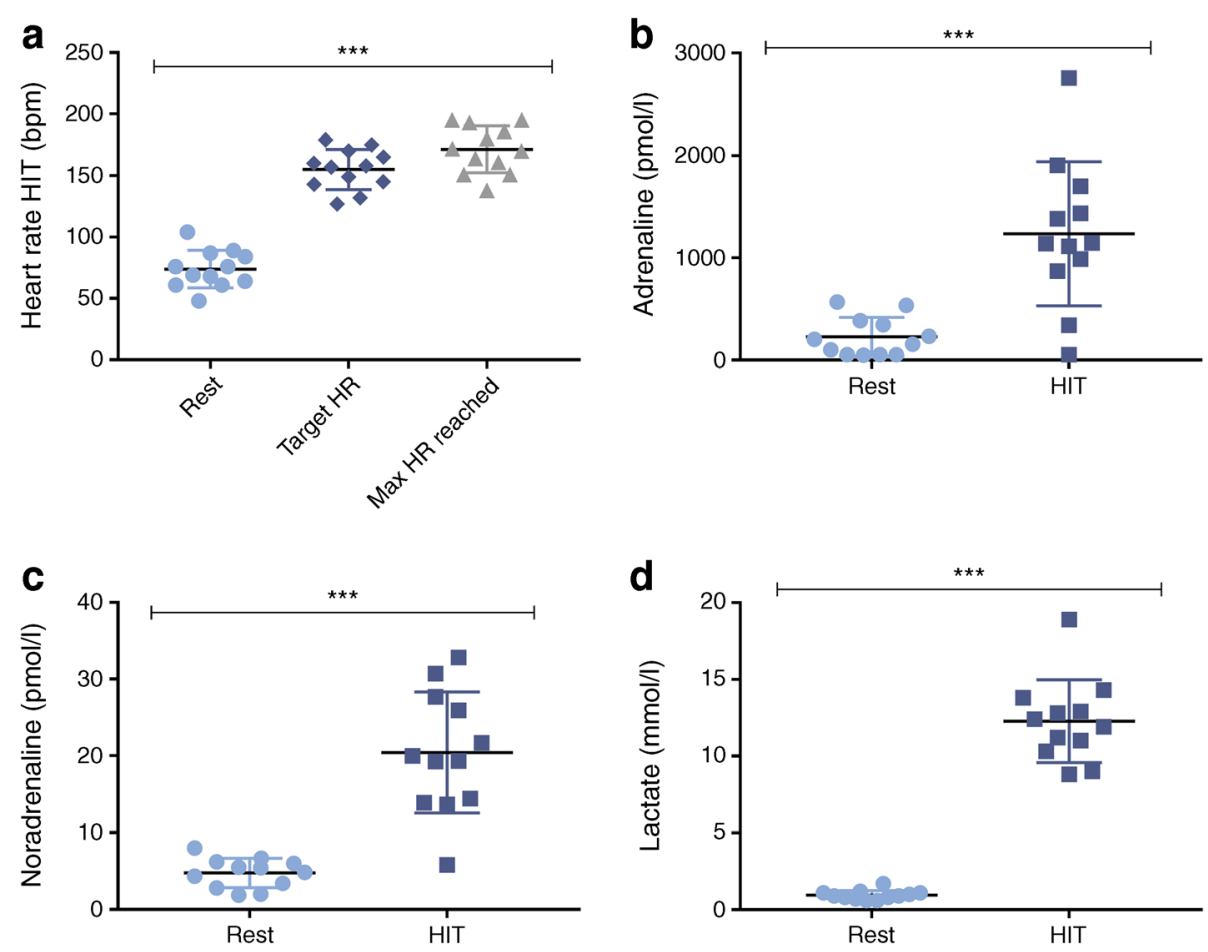

Symptomatic response Following HIT total symptom scores (where the minimal possible score is 11) measured during subsequent hypoglycaemia were significantly increased (Mean [SEM] EHS score: 24.25 [2.96] vs 27.5 [3.9], respectively, rest vs HIT, $p<0.05$; Fig. $5 \mathrm{c}$ ). However, of the EHS subcategories, while there was a numerically greater autonomic symptom score (11.75 [1.50] vs 12.83 [1.74], rest vs HIT, respectively, $p=0.144]$ and neuroglycopenic symptom score (9.75 [1.46] vs 11.42 [2.11], respectively, $p=0.174)$ in response to hypoglycaemia, these did not achieve significance.

Cognitive response We found that the 4CRT during hypoglycaemia was significantly prolonged following HIT (mean [SEM] 591.8 [22.5] vs 659.9 [39.9] ms; rest vs HIT, respectively, $p<0.01$; Fig. 5 d). There was no difference in the DSST during hypoglycaemia following rest or HIT.

\section{Discussion}

Habituation is a form of adaptive learning where the response to a stimulus is reduced or ceases altogether following repeated exposure to the stimulus. This is often considered in the context of innate behaviours $[14,15]$. The gill-withdrawal (sensory-motor) reflex in Aplysia [31] is a classic model of habituation. When the gill-withdrawal reflex is repeatedly induced by a tactile external stimulus to the siphon, the scale of the response is markedly diminished (habituation). Hypoglycaemia can be considered an internal sensory stimulus, which leads in turn to a reflex counterregulatory (motor) response [32]. If organisms adapt to repeated hypoglycaemia through habituation then it would be predicted that repeated exposure to hypoglycaemia would lead to a markedly diminished response. This is indeed the case as has been shown in humans [9, 10] and rodents [33], and as with a habituated
Fig. 4 Hyperinsulinaemichypoglycaemic clamp profiles following HIT and rest (control). (a) Plasma glucose level. (b) Glucose infusion rate. $n=12$; values are the mean $\pm \mathrm{SEM}$. GIR, glucose infusion rate
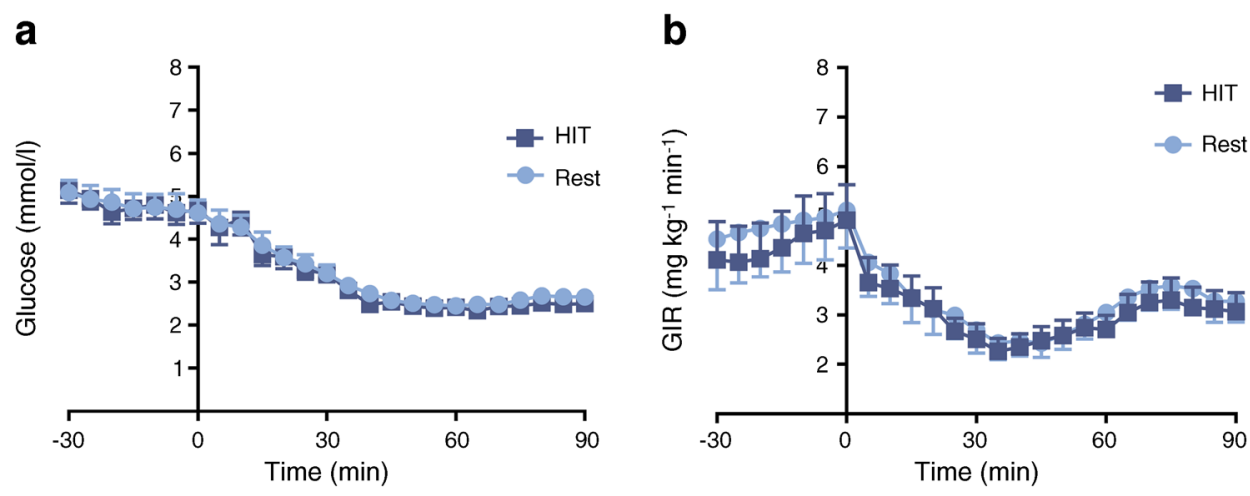
Fig. 5 Acute exposure to HIT improves defective

counterregulatory response. Plasma adrenaline (a), glucagon (b), total EHS score (c) and 4CRT (d) during the hyperinsulinaemichypoglycaemic clamp study. $n=$ 12 ; values are the mean \pm SEM. ${ }^{*} p<0.05,{ }^{*} p<<0.01$ using the generalised estimate equation
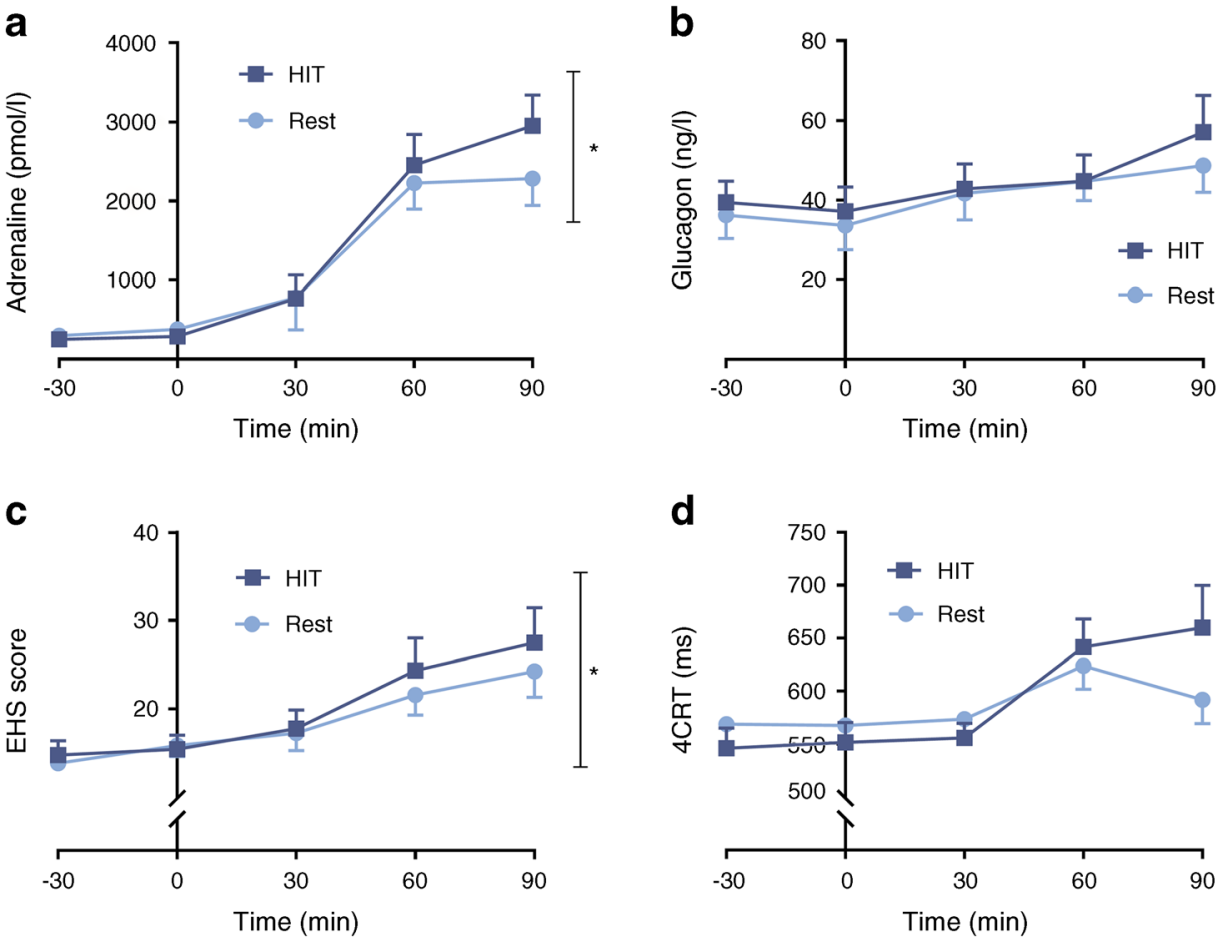

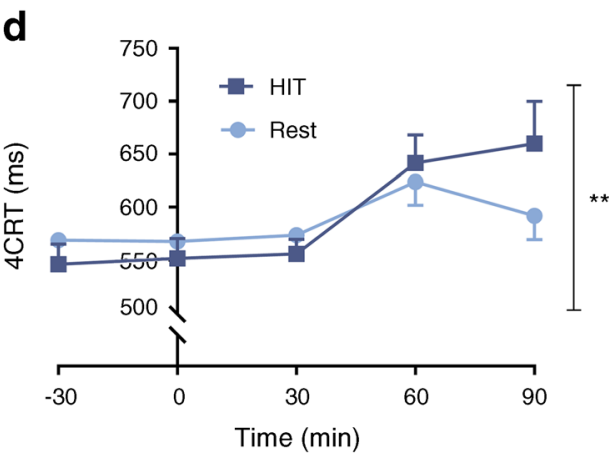

response, we see widespread and progressive suppression of physiological (e.g. suppressed counterregulatory hormonal and symptomatic responses), behavioural (e.g. reduced drive to feed, impairment when driving [17]) and psychological (e.g. reduced anxiety) responses to hypoglycaemia as a result of repeated exposure [16]. Furthermore, a cardinal feature of the habituated gill-withdrawal reflex in Aplysia is that it can then be restored, at least temporarily, by applying a strong tactile stimulus to another part of the animal (dishabituation) [31]. Our present findings, supported by our previous work in rodents [19], that hormonal, symptom and cognitive responses to hypoglycaemia can be at least partially restored following a single episode of HIT in individuals with long-standing type 1 diabetes who have been exposed to multiple episodes of hypoglycaemia, therefore provides robust support for the hypothesis that IAH develops through habituation to recurrent hypoglycaemia.

There are nine proposed criteria that define habituation [15]. As described, criterion 1: 'Given that a particular stimulus elicits a response, repeated applications of that stimulus result in a decreased response (habituation)' [15], is characteristic of recurrent hypoglycaemia $[9,10]$. Similarly, criterion 2: 'If the stimulus is withheld, the response tends to recover over time (spontaneous recovery)' [15], and we know that hypoglycaemia avoidance can lead to recovery of counterregulatory responses to subsequent hypoglycaemia $[13,34]$. In this study, we have addressed criterion 8: 'Presentation of another (usually strong) stimulus results in recovery of the habituated response (dishabituation)', and have shown that it is possible, at least temporarily, to recover hypoglycaemia counterregulation.
Interestingly, criterion 7: 'Habituation of response to a given stimulus exhibits stimulus generalization to other stimuli', may explain why our results differ from previous work suggesting that exercise can further suppress counterregulatory responses to hypoglycaemia $[35,36]$. It is possible that the mild-moderate exercise regimen, leads through stimulus 'generalisation', to suppression of the subsequent counterregulatory response to insulin-induced hypoglycaemia. The key differentiating feature between the studies being the intensity of the different stimulus.

Habituation is considered an adaptive survival response to repeated exposure of a physiological stressor in order to develop a degree of tolerance of that specific stressor. In contrast, the physiological response to repeated hypoglycaemia is currently considered maladaptive and is often referred to as hypoglycaemia-associated autonomic failure (HAAF), as originally proposed by Cryer [37]. The term HAAF is, however, misleading because, as mentioned, the suppressed counterregulatory response can be reversed in many people through strict avoidance of hypoglycaemia [13, 34]. This means the autonomic system does not 'fail', and instead is more likely to be adapting to the presence or absence of repeated hypoglycaemia. Pre-clinical studies are supportive of the habituation hypothesis in that recurrent hypoglycaemia triggers a series of changes at a cellular level that act to induce tolerance, facilitating organisms to cope better when subsequently deprived of energy. For example, prior recurrent hypoglycaemia protects against neuronal death during subsequent very severe hypoglycaemia [38]. Glucocorticoids [39] and urocortin [40], which are key to neuroprotection and initiation of adaptive cellular mechanisms [41], are also integral to 
the organism's response to recurrent hypoglycaemia. Therefore, these systematic and cellular responses to recurrent hypoglycaemia also point to IAH developing as an adaptive, habituated response to repeated hypoglycaemic stress.

In the current study we employed HIT as the novel dishabituating stimulus. We confirmed the effectiveness of the HIT intervention as a novel stimulus by demonstrating that it evoked a marked rise in heart rate, adrenaline and lactate. The day following the HIT (or rest) intervention, participants underwent a controlled hypoglycaemia study where we demonstrated significant increases induced by preceding HIT in the hormonal counterregulatory response. There was also, and of relevance in clinical practice, an increase in total symptom scores (EHS [27]) in response to matched hypoglycaemia following HIT, indicating participants were more aware of hypoglycaemia. Finally, the 4CRT was prolonged during hypoglycaemia following HIT. Choice reaction time is used to examine psychomotor and attentional focus. Acute hypoglycaemia markedly impairs performance in almost all aspects of executive function in adults with type 1 diabetes, with time taken to complete cognitive testing taking considerably longer [42, 43]. Zammitt et al have shown that 4CRT is also prolonged during experimental hypoglycaemia in people with type 1 diabetes and intact awareness, but not in those with IAH [44]. Our finding of a significant slowing of 4CRT during hypoglycaemia in participants with IAH following HIT is therefore indicative that dishabituation has, at least partially, restored counterregulatory hormonal, symptom and cognitive responses towards that seen in individuals with intact awareness.

There are a number of limitations to our study. Although the hyperinsulinaemic-hypoglycaemic clamp is the gold standard technique for assessing counterregulatory responses to hypoglycaemia [25], the studies are conducted under strictly controlled conditions in a laboratory and, as such, the experience of hypoglycaemia may not fully reflect its real-world experience. We also used three different hypoglycaemia awareness questionnaires $[4,20,21]$, which were not concordant for all participants. However, 11/12 participants scored 2 or 3 on the DAFNE hypoglycaemia awareness rating and these individuals show a fourfold increased risk of severe hypoglycaemia [21], while 10/12 participants had a modified Clarke score $\geq 4$, which carries a 4.6 -fold increased risk of severe hypoglycaemia [45].

Overall, the current study supports the hypothesis that IAH in type 1 diabetes develops as a result of habituation. This is not firmly established as additional studies will need to be performed to test the other features of habituation. However, consistent with our hypothesis, we have demonstrated that the introduction of HIT as a dishabituating stimulus, in people with long-standing type 1 diabetes and IAH, acutely restores a broad array of physiological, symptomatic and cognitive responses during subsequent experimental hypoglycaemia. If this effect is maintained following a more prolonged intervention with HIT, it may offer a novel, therapeutically attractive strategy to improve and restore hypoglycaemia awareness in people with type 1 diabetes and reduce the risk of severe hypoglycaemia.

Acknowledgements Some of the data from this work were presented as an abstract at the 55th Annual Meeting of the EASD, Barcelona, 2019. The authors would like to thank G. Kennedy and L. McFarlane, Biomarker Core Laboratory, University of Dundee, Scotland, and E. Dow at Ninewells Hospital and Medical School, Dundee, Scotland, for their advice and assistance with sample analysis. The authors would also like to thank Clinical Research Nurses: H. Loftus, L. Cabrelli and G. Wilkie, Ninewells Hospital and Medical School, Dundee, Scotland, for their assistance conducting the experiments.

Data availability Data are available on request from the corresponding author.

Funding CMF was awarded the Sir George Alberti Fellowship by Diabetes UK to carry out this research (17/0005591). Funding was also provided by the JDRF (3-SRA-2017-485-S-B).

Duality of interest The authors declare that there is no duality of interest associated with this manuscript.

Contribution statement CMF contributed to research design, conducted all experiments, acquired and analysed data and drafted the manuscript. $\mathrm{SMH}, \mathrm{ADM}, \mathrm{DW}, \mathrm{PF}$ and TJ contributed to research design, analysis of data and revision of the manuscript. RJM contributed to research design, data analysis and drafted the manuscript. All authors approved the final version of the manuscript to be published. RJM is responsible for the integrity of the work as a whole.

Open Access This article is licensed under a Creative Commons Attribution 4.0 International License, which permits use, sharing, adaptation, distribution and reproduction in any medium or format, as long as you give appropriate credit to the original author(s) and the source, provide a link to the Creative Commons licence, and indicate if changes were made. The images or other third party material in this article are included in the article's Creative Commons licence, unless indicated otherwise in a credit line to the material. If material is not included in the article's Creative Commons licence and your intended use is not permitted by statutory regulation or exceeds the permitted use, you will need to obtain permission directly from the copyright holder. To view a copy of this licence, visit http://creativecommons.org/licenses/by/4.0/.

\section{References}

1. McCoy RG, Van Houten HK, Ziegenfuss JY, Shah ND, Wermers RA, Smith SA (2012) Increased mortality of patients with diabetes reporting severe hypoglycemia. Diabetes Care 35(9):1897-1901. https://doi.org/10.2337/dc11-2054

2. Anderbro T, Gonder-Frederick L, Bolinder J et al (2015) Fear of hypoglycemia: relationship to hypoglycemic risk and psychological factors. Acta Diabetol 52(3):581-589. https://doi.org/10.1007/ s00592-014-0694-8

3. Wild D, von Maltzahn R, Brohan E, Christensen T, Clauson P, Gonder-Frederick L (2007) A critical review of the literature on fear of hypoglycemia in diabetes: implications for diabetes 
management and patient education. Patient Educ Couns 68(1):10 15. https://doi.org/10.1016/j.pec.2007.05.003

4. Gold AE, MacLeod KM, Frier BM (1994) Frequency of severe hypoglycemia in patients with type I diabetes with impaired awareness of hypoglycemia. Diabetes Care 17(7):697-703. https://doi. org/10.2337/diacare.17.7.697

5. Frier BM (2013) Impaired awareness of hypoglycaemia. In: Hypoglycaemia in clinical diabetes. Wiley, Hoboken, pp 114-144

6. Geddes J, Schopman JE, Zammitt NN, Frier BM (2008) Prevalence of impaired awareness of hypoglycaemia in adults with type 1 diabetes. Diabet Med 25(4):501-504. https://doi.org/10.1111/j. 1464-5491.2008.02413.x

7. Frier BM, Jensen MM, Chubb BD (2016) Hypoglycaemia in adults with insulin-treated diabetes in the UK: self-reported frequency and effects. Diabet Med 33(8):1125-1132. https://doi.org/10.1111/dme. 12878

8. Kristensen PL, Hansen LS, Jespersen MJ et al (2012) Insulin analogues and severe hypoglycaemia in type 1 diabetes. Diabetes Res Clin Pract 96(1):17-23. https://doi.org/10.1016/j.diabres.2011. 10.046

9. Heller SR, Cryer PE (1991) Reduced neuroendocrine and symptomatic responses to subsequent hypoglycemia after 1 episode of hypoglycemia in nondiabetic humans. Diabetes 40(2):223-226

10. Davis MR, Mellman M, Shamoon H (1992) Further defects in counterregulatory responses induced by recurrent hypoglycemia in IDDM. Diabetes 41(10):1335-1340. https://doi.org/10.2337/ diab.41.10.1335

11. Davis MR, Shamoon H (1991) Counterregulatory adaptation to recurrent hypoglycemia in normal humans. J ClinEndocrinol Metab 73(5):995-1001. https://doi.org/10.1210/jcem-73-5-995

12. Davis SN, Mann S, Galassetti P et al (2000) Effects of differing durations of antecedent hypoglycemia on counterregulatory responses to subsequent hypoglycemia in normal humans. Diabetes 49(11):1897-1903. https://doi.org/10.2337/diabetes.49. 11.1897

13. Cranston I, Lomas J, Maran A, Macdonald I, Amiel SA (1994) Restoration of hypoglycaemia awareness in patients with longduration insulin-dependent diabetes. Lancet 344(8918):283-287

14. Rankin CH, Abrams T, Barry RJ et al (2009) Habituation revisited: an updated and revised description of the behavioral characteristics of habituation. Neurobiol Learn Mem 92(2):135-138. https://doi. org/10.1016/j.nlm.2008.09.012

15. Thompson RF, Spencer WA (1966) Habituation: a model phenomenon for the study of neuronal substrates of behavior. Psychol Rev 73(1):16-43. https://doi.org/10.1037/h0022681

16. McNeilly AD, McCrimmon RJ (2018) Impaired hypoglycaemia awareness in type 1 diabetes: lessons from the lab. Diabetologia 61(4):743-750. https://doi.org/10.1007/s00125-018-4548-8

17. Cox DJ, Gonder-Frederick LA, Kovatchev BP, Julian DM, Clarke WL (2000) Progressive hypoglycemia's impact on driving simulation performance. Occurrence, awareness and correction. Diabetes Care 23(2):163-170. https://doi.org/10.2337/diacare.23.2.163

18. McNay EC, Williamson A, McCrimmon RJ, Sherwin RS (2006) Cognitive and neural hippocampal effects of long-term moderate recurrent hypoglycemia. Diabetes 55(4):1088-1095. https://doi. org/10.2337/diabetes.55.04.06.db05-1314

19. McNeilly AD, Gallagher JR, Huang JT, Ashford ML, McCrimmon RJ (2017) High intensity exercise as a dishabituating stimulus restores counterregulatory responses in recurrently hypoglycemic rodents. Diabetes. https://doi.org/10.2337/db16-1533

20. Clarke WL, Cox DJ, Gonder-Frederick LA, Julian D, Schlundt D, Polonsky W (1995) Reduced awareness of hypoglycemia in adults with IDDM. A prospective study of hypoglycemic frequency and associated symptoms. Diabetes Care 18(4):517-522. https://doi. org/10.2337/diacare.18.4.517
21. Hopkins D, Lawrence I, Mansell P et al (2012) Improved biomedical and psychological outcomes 1 year after structured education in flexible insulin therapy for people with type 1 diabetes: the U.K. DAFNE experience. Diabetes Care 35(8):1638-1642. https://doi. org/10.2337/dc11-1579

22. Araujo CG, Scharhag J (2016) Athlete: a working definition for medical and health sciences research. Scand J Med Sci Sports 26(1):4-7. https://doi.org/10.1111/sms.12632

23. Campbell WW, Kraus WE, Powell KE et al (2019) High-intensity interval training for cardiometabolic disease prevention. Med Sci Sports Exerc 51(6):1220-1226. https://doi.org/10.1249/mss. 0000000000001934

24. Riddell MC, Gallen IW, Smart CE et al (2017) Exercise management in type 1 diabetes: a consensus statement. Lancet Diabetes Endocrinol 5(5):377-390. https://doi.org/10.1016/s2213-8587(17) 30014-1

25. DeFronzo RA, Tobin JD, Andres R (1979) Glucose clamp technique: a method for quantifying insulin secretion and resistance. Am J Phys 237(3):E214-E223. https://doi.org/10.1152/ajpendo. 1979.237.3.E214

26. International Hypoglycaemia Study (2017) Glucose concentrations of less than $3.0 \mathrm{mmol} / \mathrm{l}(54 \mathrm{mg} / \mathrm{dl})$ should be reported in clinical trials: a joint position statement of the American Diabetes Association and the European Association for the Study of Diabetes. Diabetologia 60(1):3-6. https://doi.org/10.1007/s00125016-4146-6

27. Deary IJ, Hepburn DA, MacLeod KM, Frier BM (1993) Partitioning the symptoms of hypoglycaemia using multi-sample confirmatory factor analysis. Diabetologia 36(8):771-777. https:// doi.org/10.1007/bf00401150

28. Wechsler D (1981) Manual for the Wechsler adult intelligence scale revised. Psychological Corp, New York

29. Deary IJ, Liewald D, Nissan J (2011) A free, easy-to-use, computerbased simple and four-choice reaction time programme: the DearyLiewald reaction time task. Behav Res Methods 43(1):258-268. https://doi.org/10.3758/s13428-010-0024-1

30. George PS, Tavendale R, Palmer CN, McCrimmon RJ (2015) Diazoxide improves hormonal counterregulatory responses to acute hypoglycemia in long-standing type 1 diabetes. Diabetes 64(6): 2234-2241. https://doi.org/10.2337/db14-1539

31. Pinsker H, Kupfermann I, Castellucci V, Kandel E (1970) Habituation and dishabituation of the gill-withdrawal reflex in Aplysia. Science 167(3926):1740-1742. https://doi.org/10.1126/ science.167.3926.1740

32. Watts AG, Donovan CM (2010) Sweet talk in the brain: glucosensing, neural networks, and hypoglycemic counterregulation. Front Neuroendocrinol 31(1):32-43. https:// doi.org/10.1016/j.yfrne.2009.10.006

33. Beall C, Haythorne E, Fan X et al (2013) Continuous hypothalamic $\mathrm{K}_{\text {ATP }}$ activation blunts glucose counter-regulation in vivo in rats and suppresses $\mathrm{K}_{\mathrm{ATP}}$ conductance in vitro. Diabetologia 56(9): 2088-2092. https://doi.org/10.1007/s00125-013-2970-5

34. Fanelli CG, Epifano L, Rambotti AM et al (1993) Meticulous prevention of hypoglycemia normalizes the glycemic thresholds and magnitude of most of neuroendocrine responses to, symptoms of, and cognitive function during hypoglycemia in intensively treated patients with short-term IDDM. Diabetes 42(11):1683-1689

35. Sandoval DA, Guy DL, Richardson MA, Ertl AC, Davis SN (2006) Acute, same-day effects of antecedent exercise on counterregulatory responses to subsequent hypoglycemia in type 1 diabetes mellitus. Am J Phys Endocrinol Metab 290(6):E1331E1338. https://doi.org/10.1152/ajpendo.00283.2005

36. Sandoval DA, Guy DL, Richardson MA, Ertl AC, Davis SN (2004) Effects of low and moderate antecedent exercise on counterregulatory responses to subsequent hypoglycemia in type 
1 diabetes. Diabetes 53(7):1798-1806. https://doi.org/10.2337/ diabetes.53.7.1798

37. Cryer PE (1994) Banting Lecture. Hypoglycemia: the limiting factor in the management of IDDM. Diabetes 43(11):1378-1389

38. Puente EC, Silverstein J, Bree AJ et al (2010) Recurrent moderate hypoglycemia ameliorates brain damage and cognitive dysfunction induced by severe hypoglycemia. Diabetes 59(4):1055-1062. https://doi.org/10.2337/db09-1495

39. Sandoval DA, Ping L, Neill AR, Morrey S, Davis SN (2003) Cortisol acts through central mechanisms to blunt counterregulatory responses to hypoglycemia in conscious rats. Diabetes 52(9):2198-2204. https://doi.org/10.2337/diabetes.52.9. 2198

40. McCrimmon RJ, Song Z, Cheng H et al (2006) Corticotrophinreleasing factor receptors within the ventromedial hypothalamus regulate hypoglycemia-induced hormonal counterregulation. J Clin Investig 116:1723-1730

41. Grissom N, Bhatnagar S (2009) Habituation to repeated stress: get used to it. Neurobiol Learn Mem 92(2):215-224. https://doi.org/10. 1016/j.nlm.2008.07.001
42. Graveling AJ, Deary IJ, Frier BM (2013) Acute hypoglycemia impairs executive cognitive function in adults with and without type 1 diabetes. Diabetes Care 36(10):3240-3246. https://doi.org/ $10.2337 / \mathrm{dc} 13-0194$

43. Rosenthal JM, Amiel SA, Yaguez L et al (2001) The effect of acute hypoglycemia on brain function and activation: a functional magnetic resonance imaging study. Diabetes 50(7):1618-1626. https://doi.org/10.2337/diabetes.50.7.1618

44. Zammitt NN, Warren RE, Deary IJ, Frier BM (2008) Delayed recovery of cognitive function following hypoglycemia in adults with type 1 diabetes: effect of impaired awareness of hypoglycemia. Diabetes 57(3):732-736. https://doi.org/10.2337/db07-0695

45. Lin YK, Hung M, Sharma A et al (2019) Impaired awareness of hypoglycemia continues to be a risk factor for severe hypoglycemia despite the use of continuous glucose monitoring system in type 1 diabetes. Endocr Pract 25(6):517-525. https://doi.org/10.4158/ep2018-0527

Publisher's note Springer Nature remains neutral with regard to jurisdictional claims in published maps and institutional affiliations. 